\title{
Protoplasmic Astrocytoma
}

National Cancer Institute

\section{Source}

National Cancer Institute. Protoplasmic Astrocytoma. NCI Thesaurus. Code C4320.

A rare variant of diffuse astrocytoma. It is predominantly composed of neoplastic astrocytes showing a small cell body with few, flaccid processes with a low content of glial filaments and scant GFAP expression. This lesion is not well defined and is considered by some authors as an occasional histopathological feature rather than a reproducibly identifiable variant. When occurring in children, this neoplasm may be difficult to separate from pilocytic juvenile astrocytoma. (Adapted from WHO) 